\title{
A Study on Lump Solutions to a Generalized Hirota-Satsuma-Ito Equation in (2+1)-Dimensions
}

\author{
Wen-Xiu Ma $\mathbb{D},{ }^{1,2,3,4,5,6}$ Jie $^{\mathrm{Li}}{ }^{7}$ and Chaudry Masood Khalique $\mathbb{B}^{6}$ \\ ${ }^{1}$ Department of Mathematics, Zhejiang Normal University, Jinhua 321004, Zhejiang, China \\ ${ }^{2}$ Department of Mathematics, King Abdulaziz University, Jeddah, Saudi Arabia \\ ${ }^{3}$ Department of Mathematics and Statistics, University of South Florida, Tampa, FL 33620, USA \\ ${ }^{4}$ College of Mathematics and Physics, Shanghai University of Electric Power, Shanghai 200090, China \\ ${ }^{5}$ College of Mathematics and Systems Science, Shandong University of Science and Technology, Qingdao 266590, Shandong, China \\ ${ }^{6}$ International Institute for Symmetry Analysis and Mathematical Modelling, Department of Mathematical Sciences, \\ North-West University, Mafikeng Campus, Mmabatho 2735, South Africa \\ ${ }^{7}$ Jining No. 1 People's Hospital, Shandong, Jining 272011, China
}

Correspondence should be addressed to Wen-Xiu Ma; mawx@cas.usf.edu

Received 9 August 2018; Accepted 25 October 2018; Published 2 December 2018

Academic Editor: Chittaranjan Hens

Copyright (C) 2018 Wen-Xiu Ma et al. This is an open access article distributed under the Creative Commons Attribution License, which permits unrestricted use, distribution, and reproduction in any medium, provided the original work is properly cited.

\begin{abstract}
The Hirota-Satsuma-Ito equation in (2+1)-dimensions passes the three-soliton test. This paper aims to generalize this equation to a new one which still has abundant interesting solution structures. Based on the Hirota bilinear formulation, a symbolic computation with a new class of Hirota-Satsuma-Ito type equations involving general second-order derivative terms is conducted to require having lump solutions. Explicit expressions for lump solutions are successfully presented in terms of coefficients in a generalized Hirota-Satsuma-Ito equation. Three-dimensional plots and contour plots of a special presented lump solution are made to shed light on the characteristic of the resulting lump solutions.
\end{abstract}

\section{Introduction}

In the classical theory of differential equations, the main question is to study the existence of solutions to given equations, including many nonlinear equations describing real-world problems. Cauchy problems are to deal with the existence, uniqueness, and stability of solutions satisfying initial data. Laplace's method is developed for solving Cauchy problems for linear ordinary differential equations and the Fourier transform method for linear partial differential equations. In modern soliton theory, the isomonodromic transform method and the inverse scattering transform method have been designed to solve Cauchy problems for nonlinear ordinary and partial differential equations [13]. Explicitly solvable differential equations include various constant-coefficient and linear differential equations, but it is extremely difficult to compute exact solutions to variablecoefficient or nonlinear equations.
However, the Hirota bilinear method provides us with a working approach to soliton solutions, historically found for nonlinear integrable equations $[4,5]$. Soliton solutions are analytic ones exponentially localized in all directions in space and time. Let a polynomial $P$ determine a Hirota bilinear differential equation:

$$
P\left(D_{x}, D_{y}, D_{t}\right) f \cdot f=0,
$$

in (2+1)-dimensions, where $D_{x}, D_{y}$, and $D_{t}$ are Hirota's bilinear derivatives. The corresponding partial differential equation with a dependent variable $u$ is determined usually by one of the logarithmic transformations: $u=2(\ln f)_{x}$ and $u=2(\ln f)_{x x}$. Within the Hirota bilinear formulation, soliton solutions are expressed through

$$
f=\sum_{\mu=0,1} \exp \left(\sum_{i=1}^{N} \mu_{i} \xi_{i}+\sum_{i<j} \mu_{i} \mu_{j} a_{i j}\right),
$$


where $\sum_{\mu=0,1}$ means the sum over all possibilities for $\mu_{1}$, $\mu_{2}, \ldots, \mu_{N}$ taking either 0 or 1 , and the wave variables and the phase shifts are defined by

$$
\xi_{i}=k_{i} x+l_{i} y-\omega_{i} t+\xi_{i, 0}, \quad 1 \leq i \leq N
$$

and

$$
\mathrm{e}^{a_{i j}}=-\frac{P\left(k_{i}-k_{j}, l_{i}-l_{j}, \omega_{j}-\omega_{i}\right)}{P\left(k_{i}+k_{j}, l_{i}+l_{j}, \omega_{j}+\omega_{i}\right)}, \quad 1 \leq i<j \leq N,
$$

in which $k_{i}, l_{i}$, and $\omega_{i}, 1 \leq i \leq N$, satisfy the corresponding dispersion relation and $\xi_{i, 0}, 1 \leq i \leq N$, are arbitrary phase shifts.

Lump solutions are a class of analytic rational solutions which are localized in all directions in space, originated from solving integrable equations in $(2+1)$-dimensions (see, e.g., [6-8]). Taking long wave limits of $N$-soliton solutions can generate special lumps [9]. Many integrable equations in $(2+1)$-dimensions exhibit the remarkable richness of lump solutions (see, e.g., $[6,7])$. Such equations contain the KPI equation [10], whose special lump solutions have been derived from $N$-soliton solutions [11], the three-dimensional three-wave resonant interaction [12], the BKP equation [13, 14], the Davey-Stewartson equation II [9], the Ishimori-I equation [15], and the KP equation with a self-consistent source [16]. An important step in the process of getting lumps is to determine positive quadratic function solutions to bilinear equations [6]. Then, through the mentioned logarithmic transformations, we present lump solutions to nonlinear equations (see, e.g., [6] for the case of Hirota bilinear equations and [7] for the case of generalized bilinear equations).

In this paper, we would like to generalize the HirotaSatsuma-Ito (HSI) equation in (2+1)-dimensions to a new one which still has abundant interesting solution structures. Hirota bilinear forms are the starting point for our discussion (see, e.g., $[6,7,17,18]$ for other equations). We will consider a general class of HSI type equations while keeping the existence of lump solutions. A general such generalized HSI equation in (2+1)-dimensions and its lump solutions will be determined through symbolic computations with Maple. For a special presented lump solution, three-dimensional plots and contour plots will be made via the Maple plot tool, to shed light on the characteristic of the presented lump solutions. A few concluding remarks will be given in the last section.

\section{Lump Solutions}

It is known that the Hirota-Satsuma shallow water wave equation [4],

$$
\begin{aligned}
& u_{t}=u_{x x t}+3 u u_{t}-3 u_{x} v_{t}-u_{x}, \\
& v_{x}=-u,
\end{aligned}
$$

has a bilinear form,

$$
\left(D_{t} D_{x}^{3}-D_{t} D_{x}-D_{x}^{2}\right) f \cdot f=0,
$$

under the logarithmic transformations $u=2(\ln f)_{x x}$ and $v=$ $2(\ln f)_{x}$. An integrable $(2+1)$-dimensional extension of the Hirota-Satsuma equation reads

$$
3\left(u_{x} u_{t}\right)_{x}+u_{x x x t}+u_{y t}+u_{x x}=0,
$$

which passes the Hirota three-soliton test [19], and has a bilinear form under the logarithmic transformation $u=$ $2(\ln f)_{x}$ :

$$
\left(D_{x}^{3} D_{t}+D_{y} D_{t}+D_{x}^{2}\right) f \cdot f=0 .
$$

Equation (7) is called the Hirota-Satsuma-Ito (HSI) equation in (2+1)-dimensions [19]. We would like to add three terms to generalize the abovementioned HSI equation to a new one which still possesses abundant interesting solution structures:

$$
\begin{aligned}
P(u)= & 3\left(u_{x} u_{t}\right)_{x}+u_{x x x t}+\delta_{1} u_{y t}+\delta_{2} u_{x x}+\delta_{3} u_{x y} \\
& +\delta_{4} u_{x t}+\delta_{5} u_{y y}=0 .
\end{aligned}
$$

This generalized HSI equation has a bilinear form under the logarithmic transformation $u=2(\ln f)_{x}$ :

$$
\begin{aligned}
& B(f)=\left(D_{x}^{3} D_{t}+\delta_{1} D_{y} D_{t}+\delta_{2} D_{x}^{2}+\delta_{3} D_{x} D_{y}\right. \\
& \left.\quad+\delta_{4} D_{x} D_{t}+\delta_{5} D_{y}^{2}\right) f \cdot f=0 .
\end{aligned}
$$

Precisely, under $u=2(\ln f)_{x}$, we have the relation $P(u)=$ $\left(B(f) / f^{2}\right)_{x}$. In what follows, we would like to determine lump solutions to the gHSI equation in (2+1)-dimensions (9), through symbolic computations with Maple.

We start to search for positive quadratic solutions to the gHSI bilinear equation (10) to generate lump solutions to the gHSI equation (9):

$$
\begin{aligned}
f= & \left(a_{1} x+a_{2} y+a_{3} t+a_{4}\right)^{2}+\left(a_{5} x+a_{6} y+a_{7} t+a_{8}\right)^{2} \\
& +a_{9} .
\end{aligned}
$$

Plugging this function into the gHSI bilinear equation (10) generates a system of nonlinear algebraic equations on the parameters $a_{i}, 1 \leq i \leq 9$. Conducting direct symbolic computation to solve this system gives a set of solutions for the parameters where

$$
\begin{aligned}
& a_{3}=-\frac{b_{1}}{\left(a_{1} \delta_{4}+a_{2} \delta_{1}\right)^{2}+\left(a_{5} \delta_{4}+a_{6} \delta_{1}\right)^{2}}, \\
& a_{7}=-\frac{b_{2}}{\left(a_{1} \delta_{4}+a_{2} \delta_{1}\right)^{2}+\left(a_{5} \delta_{4}+a_{6} \delta_{1}\right)^{2}}, \\
& a_{9}=\frac{3\left(a_{1}^{2}+a_{5}^{2}\right) b_{3}}{\left(a_{1} a_{6}-a_{2} a_{5}\right)^{2}\left(\delta_{1}^{2} \delta_{2}-\delta_{1} \delta_{3} \delta_{4}+\delta_{4}^{2} \delta_{5}\right)},
\end{aligned}
$$


and all other $a_{i}$ 's are arbitrary. The involved three constants are defined as follows:

$$
\begin{aligned}
b_{1}= & {\left[\left(a_{1}^{2} a_{2}+2 a_{1} a_{5} a_{6}-a_{2} a_{5}^{2}\right) \delta_{2}+a_{1}\left(a_{2}^{2}+a_{6}^{2}\right) \delta_{3}\right.} \\
& \left.+a_{2}\left(a_{2}^{2}+a_{6}^{2}\right) \delta_{5}\right] \delta_{1}+\left[a_{1}\left(a_{1}^{2}+a_{5}^{2}\right) \delta_{2}\right. \\
& \left.+a_{2}\left(a_{1}^{2}+a_{5}^{2}\right) \delta_{3}+\left(a_{1} a_{2}^{2}+2 a_{2} a_{5} a_{6}-a_{1} a_{6}^{2}\right) \delta_{5}\right] \delta_{4}, \\
b_{2}= & {\left[\left(-a_{1}^{2} a_{6}+2 a_{1} a_{2} a_{5}+a_{5}^{2} a_{6}\right) \delta_{2}+a_{5}\left(a_{2}^{2}+a_{6}^{2}\right) \delta_{3}\right.} \\
& \left.+a_{6}\left(a_{2}^{2}+a_{6}^{2}\right) \delta_{5}\right] \delta_{1}+\left[a_{5}\left(a_{1}^{2}+a_{5}^{2}\right) \delta_{2}\right. \\
& \left.+a_{6}\left(a_{1}^{2}+a_{5}^{2}\right) \delta_{3}+\left(-a_{2}^{2} a_{5}+2 a_{1} a_{2} a_{6}+a_{5} a_{6}^{2}\right) \delta_{5}\right] \\
& \cdot \delta_{4}, \\
b_{3} & =\left(a_{1}^{2}+a_{5}^{2}\right)\left(a_{1} a_{2}+a_{5} a_{6}\right)\left(\delta_{1} \delta_{2}+\delta_{3} \delta_{4}\right)+\left(a_{1}^{2}\right. \\
& \left.+a_{5}^{2}\right)\left(a_{2}^{2}+a_{6}^{2}\right) \delta_{1} \delta_{3}+\left(a_{1}^{2}+a_{5}^{2}\right)^{2} \delta_{2} \delta_{4}+\left(a_{2}^{2}+a_{6}^{2}\right) \\
& \cdot\left(a_{1} a_{2}+a_{5} a_{6}\right) \delta_{1} \delta_{5}+\left[\left(a_{1} a_{2}+a_{5} a_{6}\right)^{2}\right. \\
& \left.-\left(a_{1} a_{6}-a_{2} a_{5}\right)^{2}\right] \delta_{4} \delta_{5} .
\end{aligned}
$$

Those formulas in (12) and (13) were obtained under a simplification process with Maple.

From (12), we can easily see that it is sufficient to guarantee $f>0$ if we require

$$
\left(\delta_{1}^{2} \delta_{2}-\delta_{1} \delta_{3} \delta_{4}+\delta_{4}^{2} \delta_{5}\right) b_{3}>0
$$

and, thus, the function $f$ defined by (12) and (13) under the abovementioned condition and

$$
a_{1} a_{6}-a_{2} a_{5} \neq 0
$$

leads to lump solutions

$$
u=2(\ln f)_{x}=\frac{2 f_{x}}{f}
$$

to the gHSI equation in (2+1)-dimensions (9).

When one takes

$$
\begin{aligned}
& \delta_{1}=1, \\
& \delta_{2}=1, \\
& \delta_{3}=\delta_{4}=\delta_{5}=0,
\end{aligned}
$$

one obtains the original HSI equation in $(2+1)$-dimensions (7), and the function $f$ by (12) and (13) presents a class of lump solutions to the HSI equation (7):

$$
\begin{aligned}
u= & 2(\ln f)_{x}, \\
f= & \left(a_{1} x+a_{2} y+a_{3} t+a_{4}\right)^{2} \\
& +\left(a_{5} x+a_{6} y+a_{7} t+a_{8}\right)^{2}+a_{9},
\end{aligned}
$$

where

$$
\begin{aligned}
& a_{3}=-\frac{a_{1}^{2} a_{2}+2 a_{1} a_{5} a_{6}-a_{2} a_{5}^{2}}{a_{2}^{2}+a_{6}^{2}}, \\
& a_{7}=\frac{a_{1}^{2} a_{6}-2 a_{1} a_{2} a_{5}-a_{5}^{2} a_{6}}{a_{2}^{2}+a_{6}^{2}}, \\
& a_{9}=\frac{3\left(a_{1}^{2}+a_{5}^{2}\right)^{2}\left(a_{1} a_{2}+a_{5} a_{6}\right)}{\left(a_{1} a_{6}-a_{2} a_{5}\right)^{2}},
\end{aligned}
$$

and all other $a_{i}$ 's are arbitrary. Solving the abovementioned parameter solutions on $a_{3}$ and $a_{7}$ for $a_{2}$ and $a_{6}$ and substituting the resulting expressions for $a_{2}$ and $a_{6}$ into the formula for $a_{9}$ in (19), we get

$$
\begin{aligned}
& a_{2}=-\frac{a_{1}^{2} a_{3}+2 a_{1} a_{5} a_{7}-a_{3} a_{5}^{2}}{a_{3}^{2}+a_{7}^{2}}, \\
& a_{6}=\frac{a_{1}^{2} a_{7}-2 a_{1} a_{3} a_{5}-a_{5}^{2} a_{7}}{a_{3}^{2}+a_{7}^{2}}, \\
& a_{9}=-\frac{3\left(a_{1}^{2}+a_{5}^{2}\right)\left(a_{3}^{2}+a_{7}^{2}\right)\left(a_{1} a_{3}+a_{5} a_{7}\right)}{\left(a_{1} a_{7}-a_{3} a_{5}\right)^{2}} .
\end{aligned}
$$

It is easy to see that

$$
a_{1} a_{6}-a_{2} a_{5}=\frac{\left(a_{1}^{2}+a_{5}^{2}\right)\left(a_{1} a_{7}-a_{3} a_{5}\right)}{a_{3}^{2}+a_{7}^{2}}
$$

and, thus, the conditions of

$$
\begin{aligned}
& a_{1} a_{3}+a_{5} a_{7}<0, \\
& a_{1} a_{7}-a_{3} a_{5} \neq 0
\end{aligned}
$$

guarantee that (16) with (11) and (20) will present lump solutions to the HSI equation (7) [20].

Particularly taking

$$
\begin{aligned}
& \delta_{1}=1, \\
& \delta_{2}=1, \\
& \delta_{3}=-1, \\
& \delta_{4}=1, \\
& \delta_{5}=-1,
\end{aligned}
$$

we obtain a special gHSI equation as follows:

$$
u_{x x x t}+3\left(u_{x} u_{t}\right)_{x}+u_{y t}+u_{x x}-u_{x y}+u_{x t}-u_{y y}=0 \text {, }
$$

which has a Hirota bilinear form

$$
\begin{aligned}
& \left(D_{x}^{3} D_{t}+D_{y} D_{t}+D_{x}^{2}-D_{x} D_{y}+D_{x} D_{t}-D_{y}^{2}\right) f \cdot f \\
& \quad=0
\end{aligned}
$$



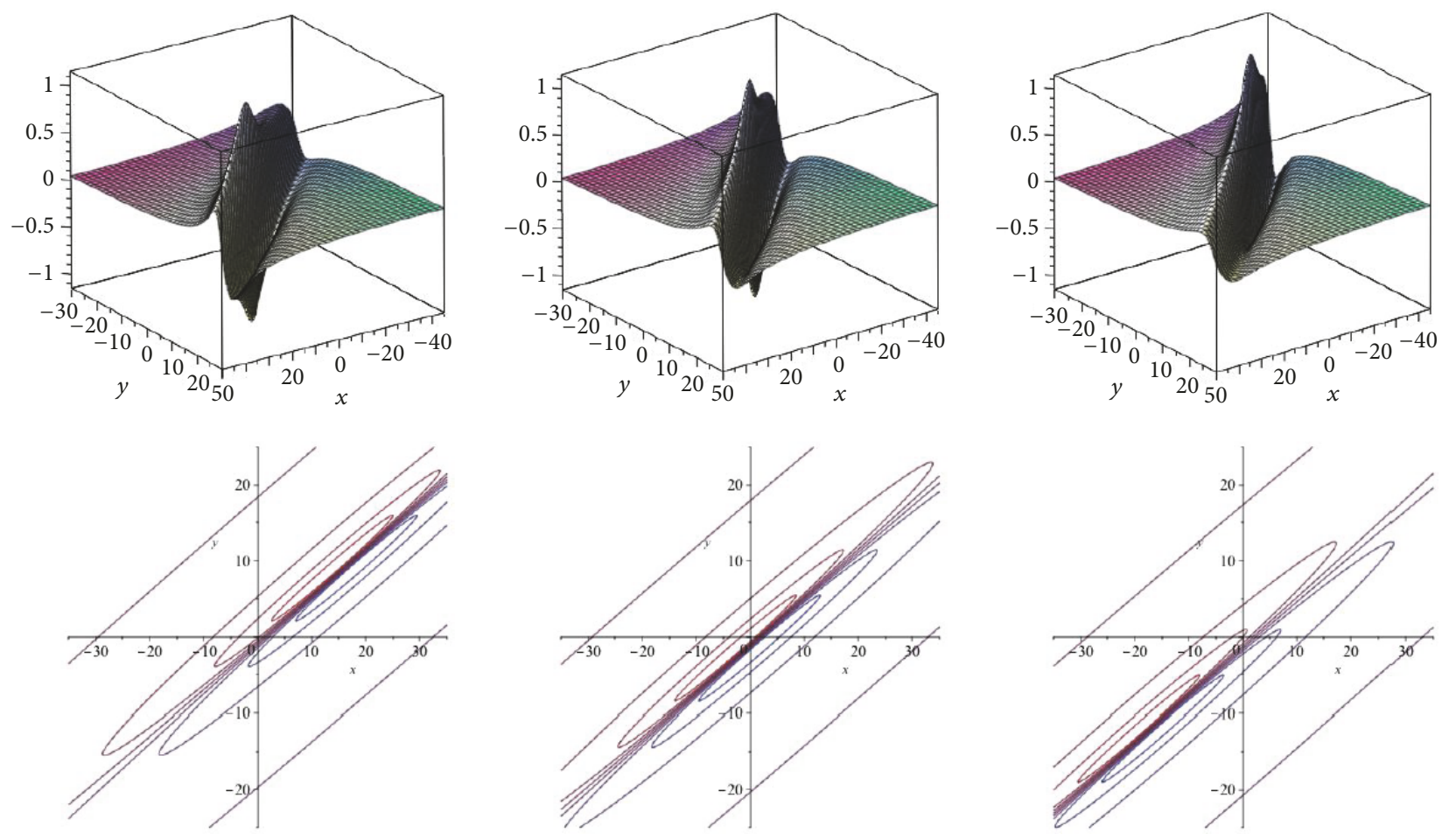

Figure 1: Profiles of $u$ when $t=0,3,6: 3$ D plots (top) and contour plots (bottom).

under the logarithmic transformation (16). Associated with

$$
\begin{aligned}
& a_{1}=1, \\
& a_{2}=-2, \\
& a_{4}=2, \\
& a_{5}=2, \\
& a_{6}=-3, \\
& a_{8}=-5,
\end{aligned}
$$

(16) with (11) and (22) present the lump solution to the special gHSI equation (24):

$u$

$$
=\frac{2(10 x-16 y-t-16)}{(x-2 y-(3 / 2) t+2)^{2}+(2 x-3 y+(1 / 2) t-5)^{2}+15} .
$$

Three three-dimensional plots and contour plots of this lump solution are made via Maple plot tools, to shed light on the characteristic of the presented lump solutions, in Figure 1.

All the exact solutions generated above add valuable insights into the existing theories on soliton solutions and dromion-type solutions, developed through various powerful solution techniques including the Hirota perturbation approach, the Riemann-Hilbert approach, the Wronskian technique, symmetry reductions, and symmetry constraints (see, e.g., [21-31]).

\section{Concluding Remarks}

We have studied a generalized (2+1)-dimensional HirotaSatsuma-Ito (HSI) equation to explore different equations which possess lump solutions, through symbolic computations with Maple. The results enrich the theory of lumps and solitons, providing a new example of $(2+1)$-dimensional nonlinear equations, which possess beautiful lump structures. Three-dimensional plots and contour plots of a specially chosen lump solution were made by using the plot tool in Maple.

Many nonlinear equations possess lump solutions, which include $(2+1)$-dimensional generalized KP, BKP, KP-Boussinesq, Sawada-Kotera, and Bogoyavlensky-Konopelchenko equations [32-36]. Some recent studies also demonstrate the strikingly high richness of lump solutions to linear partial differential equations [37] and nonlinear partial differential equations in $(2+1)$-dimensions (see, e.g., [38-41]) and (3+1)-dimensions (see, e.g., [42-48]). Diversity of lump solutions supplements exact solutions generated from different kinds of combinations (see, e.g., [49-52]) and can yield various Lie-Bäcklund symmetries, which can be used to determine conservation laws by symmetries and adjoint symmetries [53-55]. Moreover, diverse interaction solutions [35] have been exhibited for many integrable equations in (2+1)-dimensions, including lump-soliton interaction solutions (see, e.g., [56-58]) and lump-kink interaction solutions (see, e.g., [59-62]). 
We finally remark that we could add one more term to the gHSI equation (9) to formulate a more generalized HSI bilinear equation,

$$
\begin{aligned}
& \left(D_{x}^{3} D_{t}+\delta_{1} D_{y} D_{t}+\delta_{2} D_{x}^{2}+\delta_{3} D_{x} D_{y}+\delta_{4} D_{x} D_{t}\right. \\
& \left.\quad+\delta_{5} D_{y}^{2}+\delta_{6} D_{t}^{2}\right) f \cdot f=0,
\end{aligned}
$$

where $\delta_{i}, 1 \leq i \leq 6$, are all constants, but we failed to drive any lump solution to the corresponding nonlinear equation on $u=2(\ln f)_{x}$. The first term in the abovementioned bilinear equation is crucial in determining lump solutions but the last term brings the difficulty to work out lump solutions. There is no hint on how to solve any big system of resulting nonlinear algebraic equations. Nevertheless, some general considerations on the existence of lumps have been made for the Hirota bilinear case [6] and the generalized bilinear cases [7].

\section{Data Availability}

The data used to support the findings of this study are included within the article.

\section{Conflicts of Interest}

The authors declare that there are no conflicts of interest regarding the publication of this paper.

\section{Acknowledgments}

The work was supported in part by NSFC under Grants nos. 11301454, 11301331, and 11371086, NSF under Grant no. DMS1664561, the Natural Science Foundation for Colleges and Universities in Jiangsu Province (17KJB110020), Emphasis Foundation of Special Science Research on Subject Frontiers of CUMT under Grant no. 2017XKZD11, and the Distinguished Professorships by Shanghai University of Electric Power, China, and North-West University, South Africa.

\section{References}

[1] S. P. Novikov, S. V. Manakov, L. P. Pitaevskii, and V. E. Zakharov, Theory of Solitons: The Inverse Scattering Method, Plenum Press, New York, NY, USA, 1984.

[2] M. J. Ablowitz and P. A. Clarkson, Solitons, Nonlinear Evolution Equations and Inverse Scattering, Cambridge University Press, New York, NY, USA, 1991.

[3] P. G. Drazin and R. S. Johnson, Solitons: An Introduction, Cambridge University Press, Cambridge, UK, 1st edition, 1989.

[4] R. Hirota, The Direct Method in Soliton Theory, Cambridge University Press, Cambridge, UK, 2004.

[5] P. J. Caudrey, "Memories of Hirota's method: application to the reduced Maxwell-Bloch system in the early 1970s," Philosophical Transactions of the Royal Society A: Mathematical, Physical \& Engineering Sciences, vol. 369, no. 1939, pp. 1215-1227, 2011.

[6] W. X. Ma and Y. Zhou, "Lump solutions to nonlinear partial differential equations via Hirota bilinear forms," Journal of Differential Equations, vol. 264, no. 4, pp. 2633-2659, 2018.
[7] W. X. Ma, Y. Zhou, and R. Dougherty, "Lump-type solutions to nonlinear differential equations derived from generalized bilinear equations," International Journal of Modern Physics B, vol. 30, no. 28-29, 1640018, 16 pages, 2016.

[8] W. Tan, H. Dai, Z. Dai, and W. Zhong, "Emergence and space-time structure of lump solution to the $(2+1)$-dimensional generalized KP equation," Pramana-Journal of Physics, vol. 89, no. 5, 77, 7 pages, 2017.

[9] J. Satsuma and M. J. Ablowitz, "Two-dimensional lumps in nonlinear dispersive systems," Journal of Mathematical Physics, vol. 20, no. 7, pp. 1496-1503, 1979.

[10] W. X. Ma, "Lump solutions to the Kadomtsev-Petviashvili equation," Physics Letters A, vol. 379, no. 36, Article ID 23311, pp. 1975-1978, 2015.

[11] S. V. Manakov, V. E. Zakharov, L. A. Bordag, A. R. Its, and V. B. Matveev, "Two-dimensional solitons of the KadomtsevPetviashvili equation and their interaction," Physics Letters A, vol. 63, no. 3, pp. 205-206, 1977.

[12] D. J. Kaup, “The lump solutions and the Bäcklund transformation for the three-dimensional three-wave resonant interaction," Journal of Mathematical Physics, vol. 22, no. 6, pp. 11761181, 1981.

[13] C. R. Gilson and J. J. Nimmo, "Lump solutions of the BKP equation," Physics Letters A, vol. 147, no. 8-9, pp. 472-476, 1990.

[14] J. Y. Yang and W. X. Ma, "Lump solutions to the BKP equation by symbolic computation," International Journal of Modern Physics $B$, vol. 30, no. 28-29, Article ID 1640028, 7 pages, 2016.

[15] K. Imai, "Dromion and lump solutions of the Ishimori-I equation," Progress of Theoretical and Experimental Physics, vol. 98, no. 5, pp. 1013-1023, 1997.

[16] X. L. Yong, W. X. Ma, Y. H. Huang, and Y. Liu, "Lump solutions to the Kadomtsev-Petviashvili I equation with a self-consistent source," Computers \& Mathematics with Applications, vol. 75, no. 9, pp. 3414-3419, 2018.

[17] Y. Zhou and W. X. Ma, "Applications of linear superposition principle to resonant solitons and complexitons," Computers \& Mathematics with Applications, vol. 73, no. 8, pp. 1697-1706, 2017.

[18] X. Lü, W. X. Ma, Y. Zhou, and C. M. Khalique, "Rational solutions to an extended Kadomtsev-Petviashvili-like equation with symbolic computation," Computers \& Mathematics with Applications, vol. 71, no. 8, pp. 1560-1567, 2016.

[19] J. Hietarinta, "Introduction to the Hirota bilinear method," in Integrability of Nonlinear Systems, Y. Kosmann-Schwarzbach, B. Grammaticos, and K. M. Tamizhmani, Eds., pp. 95-103, Springer, Berlin, Heidelberg, 1997.

[20] Y. Zhou, S. Manukure, and W. X. Ma, "Lump and lump-soliton solutions to the Hirota-Satsuma-Ito equation," Communications in Nonlinear Science and Numerical Simulation, vol. 68, pp. 56-62, 2019.

[21] X. Lü, W. X. Ma, S. T. Chen, and C. M. Khalique, "A note on rational solutions to a Hirota-Satsuma-like equation," Applied Mathematics Letters, vol. 58, pp. 13-18, 2016.

[22] J. P. Wu and X. G. Geng, "Novel Wronskian condition and new exact solutions to a $(3+1)$-dimensional generalized KP equation," Communications in Theoretical Physics, vol. 60, no. 5, pp. 556-560, 2013.

[23] B. Dorizzi, B. Grammaticos, A. Ramani, and P. Winternitz, "Are all the equations of the Kadomtsev-Petviashvili hierarchy integrable?" Journal of Mathematical Physics, vol. 27, no. 12, pp. 2848-2852, 1986. 
[24] B. Konopelchenko and W. Strampp, "The AKNS hierarchy as symmetry constraint of the KP hierarchy," Inverse Problems, vol. 7, no. 2, pp. L17-L24, 1991.

[25] X. Y. Li, Q. L. Zhao, Y. X. Li, and H. H. Dong, "Binary bargmann symmetry constraint associated with $3 \times 3$ discrete matrix spectral problem," Journal of Nonlinear Sciences and Applications, vol. 8, no. 5, pp. 496-506, 2015.

[26] Q. L. Zhao and X. Y. Li, "A Bargmann system and the involutive solutions associated with a new 4-order lattice hierarchy," Analysis and Mathematical Physics, vol. 6, no. 3, pp. 237-254, 2016.

[27] D. S. Wang and Y. Yin, "Symmetry analysis and reductions of the two-dimensional generalized Benney system via geometric approach," Computers \& Mathematics with Applications, vol. 71, no. 3, pp. 748-757, 2016.

[28] H. Dong, Y. Zhang, and X. Zhang, "The new integrable symplectic map and the symmetry of integrable nonlinear lattice equation," Communications in Nonlinear Science and Numerical Simulation, vol. 36, pp. 354-365, 2016.

[29] X. Y. Li and Q. L. Zhao, "A new integrable symplectic map by the binary nonlinearization to the super AKNS system," Journal of Geometry and Physics, vol. 121, pp. 123-137, 2017.

[30] J. G. Liu, L. Zhou, and Y. He, "Multiple soliton solutions for the new (2+1)-dimensional Korteweg-de Vries equation by multiple exp-function method," Applied Mathematics Letters, vol. 80, pp. 71-78, 2018.

[31] W. X. Ma, "Riemann-Hilbert problems and N-soliton solutions for a coupled mKdV system," Journal of Geometry and Physics, vol. 132, pp. 45-54, 2018.

[32] W. X. Ma, Z. Qin, and X. Lü, "Lump solutions to dimensionally reduced p-gkp and p-gbkp equations," Nonlinear Dynamics, vol. 84, no. 2, pp. 923-931, 2016.

[33] X. Lü, S. T. Chen, and W. X. Ma, "Constructing lump solutions to a generalized Kadomtsev-Petviashvili-Boussinesq equation," Nonlinear Dynamics, vol. 86, no. 1, pp. 523-534, 2016.

[34] H. Q. Zhang and W. X. Ma, "Lump solutions to the $(2+1)$ dimensional Sawada-Kotera equation," Nonlinear Dynamics, vol. 87, no. 4, pp. 2305-2310, 2017.

[35] W. X. Ma, X. Yong, and H. Q. Zhang, "Diversity of interaction solutions to the (2+1)-dimensional Ito equation," Computers \& Mathematics with Applications, vol. 75, no. 1, pp. 289-295, 2018.

[36] S. T. Chen and W. X. Ma, "Lump solutions to a generalized Bogoyavlensky-Konopelchenko equation," Frontiers of Mathematics in China, vol. 13, no. 3, pp. 525-534, 2018.

[37] W. X. Ma, "Diverse lump and interaction solutions to linear PDEs in (3+1)-dimensions," East Asian J. Appl. Math, 2019.

[38] J. P. Yu and Y. L. Sun, "Study of lump solutions to dimensionally reduced generalized KP equations," Nonlinear Dynamics, vol. 87, no. 4, pp. 2755-2763, 2017.

[39] X. Zhang, Y. Chen, and Y. Zhang, "Breather, lump and soliton solutions to nonlocal KP equation," Computers \& Mathematics with Applications, vol. 74, no. 10, pp. 2341-2347, 2017.

[40] S. Manukure, Y. Zhou, and W. X. Ma, "Lump solutions to a (2+1)-dimensional extended KP equation," Computers \& Mathematics with Applications, vol. 75, no. 7, pp. 2414-2419, 2018.

[41] H. Wang, "Lump and interaction solutions to the $(2+1)$ dimensional Burgers equation," Applied Mathematics Letters, vol. 85, pp. 27-34, 2018.
[42] W. X. Ma, "Lump-type solutions to the $(3+1)$-dimensional Jimbo-Miwa equation," International Journal of Nonlinear Sciences and Numerical Simulation, vol. 17, no. 7-8, pp. 355-359, 2016.

[43] Harun-Or-Roshid and M. Z. Ali, "Lump solutions to a JimboMiwa like equation," https://arxiv.org/abs/1611.04478.

[44] Y. Zhang, H. Dong, X. Zhang, and H. Yang, "Rational solutions and lump solutions to the generalized (3+1)-dimensional shallow water-like equation," Journal of Computers \& Mathematics with Applications, vol. 73, no. 2, pp. 246-252, 2017.

[45] J. Y. Yang and W. X. Ma, “"Abundant lump-type solutions of the Jimbo-Miwa equation in (3+1)-dimensions," Computers \& Mathematics with Applications, vol. 73, no. 2, pp. 220-225, 2017.

[46] Y. Zhang, S. Sun, and H. Dong, "Hybrid Solutions of $(3+1)$ Dimensional Jimbo-Miwa Equation," Mathematical Problems in Engineering, vol. 2017, Article ID 5453941, 15 pages, 2017.

[47] Y. Zhang, Y. Liu, and X. Tang, "M-lump solutions to a (3+1)-dimensional nonlinear evolution equation," Computers \& Mathematics with Applications, vol. 76, no. 3, pp. 592-601, 2018.

[48] Y. Sun, B. Tian, X. Y. Xie, J. Chai, and H. M. Yin, "Rogue waves and lump solitons for a (3+1)-dimensional B-type KadomtsevPetviashvili equation in fluid dynamics," Wave Random Complex, vol. 28, pp. 544-552, 2018.

[49] W. X. Ma and E. G. Fan, "Linear superposition principle applying to Hirota bilinear equations," Computers \& Mathematics with Applications, vol. 61, no. 4, pp. 950-959, 2011.

[50] H. C. Zheng, W. X. Ma, and X. Gu, "Hirota bilinear equations with linear subspaces of hyperbolic and trigonometric function solutions," Applied Mathematics and Computation, vol. 220, pp. 226-234, 2013.

[51] Z. Xu, H. Chen, and Z. Da, "Rogue wave for the $(2+1)$ dimensional Kadomtsev-Petviashvili equation," Applied Mathematics Letters, vol. 37, pp. 34-38, 2014.

[52] Ö. Ünsal and W. X. Ma, "Linear superposition principle of hyperbolic and trigonometric function solutions to generalized bilinear equations," Computers \& Mathematics with Applications, vol. 71, no. 6, pp. 1242-1247, 2016.

[53] N. H. Ibragimov, "A new conservation theorem," Journal of Mathematical Analysis and Applications, vol. 333, no. 1, pp. 311328, 2007.

[54] W. X. Ma, "Conservation laws of discrete evolution equations by symmetries and adjoint symmetries," Symmetry, vol. 7, no. 2, pp. 714-725, 2015.

[55] W. X. Ma, "Conservation laws by symmetries and adjoint symmetries," Discrete and Continuous Dynamical Systems Series S, vol. 11, no. 4, pp. 707-721, 2018.

[56] J. Y. Yang and W. X. Ma, "Abundant interaction solutions of the KP equation," Nonlinear Dynamics, vol. 89, no. 2, pp. 1539-1544, 2017.

[57] J. Y. Yang, W. X. Ma, and Z. Qin, "Lump and lump-soliton solutions to the $(2+1)$-dimensional Ito equation," Analysis and Mathematical Physics, vol. 8, no. 3, pp. 427-436, 2018.

[58] J. Y. Yang, W. X. Ma, and Z. Qin, "Abundant Mixed LumpSoliton Solutions to the BKP Equation," East Asian Journal on Applied Mathematics, vol. 8, no. 2, pp. 224-232, 2018.

[59] Y. Tang, S. Tao, and Q. Guan, "Lump solitons and the interaction phenomena of them for two classes of nonlinear evolution equations," Computers \& Mathematics with Applications, vol. 72, no. 9, pp. 2334-2342, 2016.

[60] H. Q. Zhao and W. X. Ma, "Mixed lump-kink solutions to the KP equation," Computers \& Mathematics with Applications. An International Journal, vol. 74, no. 6, pp. 1399-1405, 2017. 
[61] J. B. Zhang and W. X. Ma, "Mixed lump-kink solutions to the BKP equation," Computers \& Mathematics with Applications. An International Journal, vol. 74, no. 3, pp. 591-596, 2017.

[62] T. C. Kofane, M. Fokou, A. Mohamadou, and E. Yomba, "Lump solutions and interaction phenomenon to the thirdorder nonlinear evolution equation," The European Physical Journal Plus, vol. 132, no. 11, Article ID 465, 2017. 


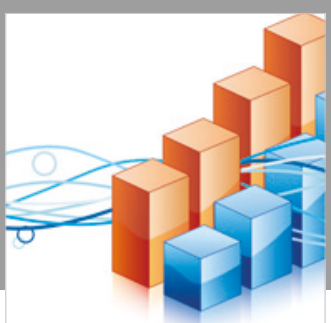

Advances in

Operations Research

\section{-n-m}
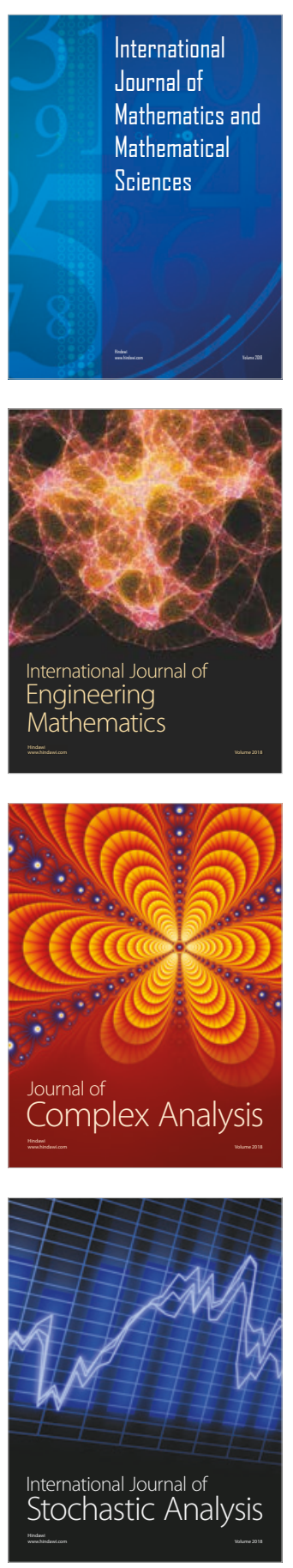
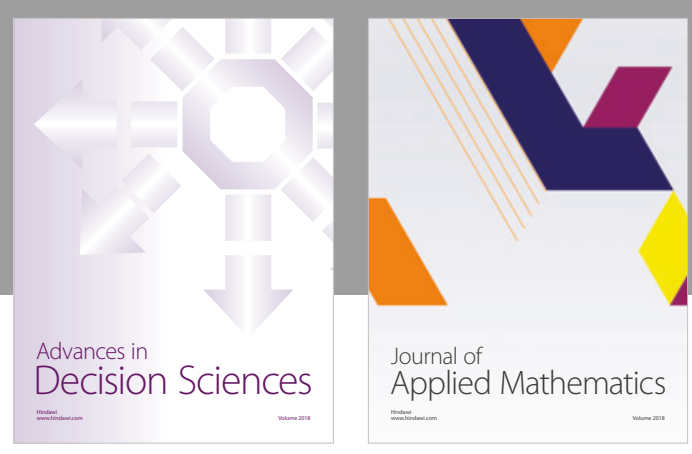

Journal of

Applied Mathematics
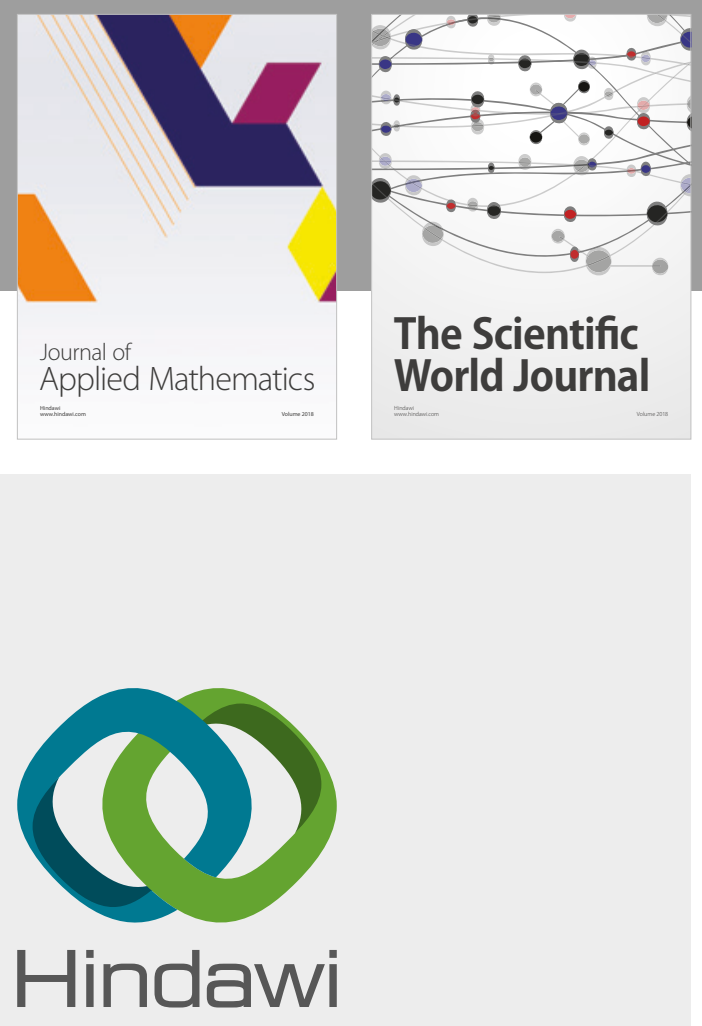

Submit your manuscripts at

www.hindawi.com

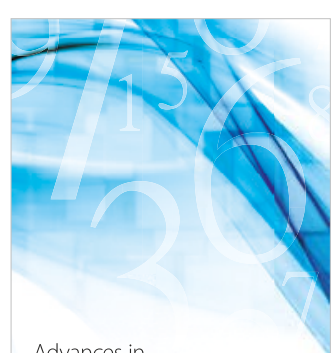

Advances in
Numerical Analysis
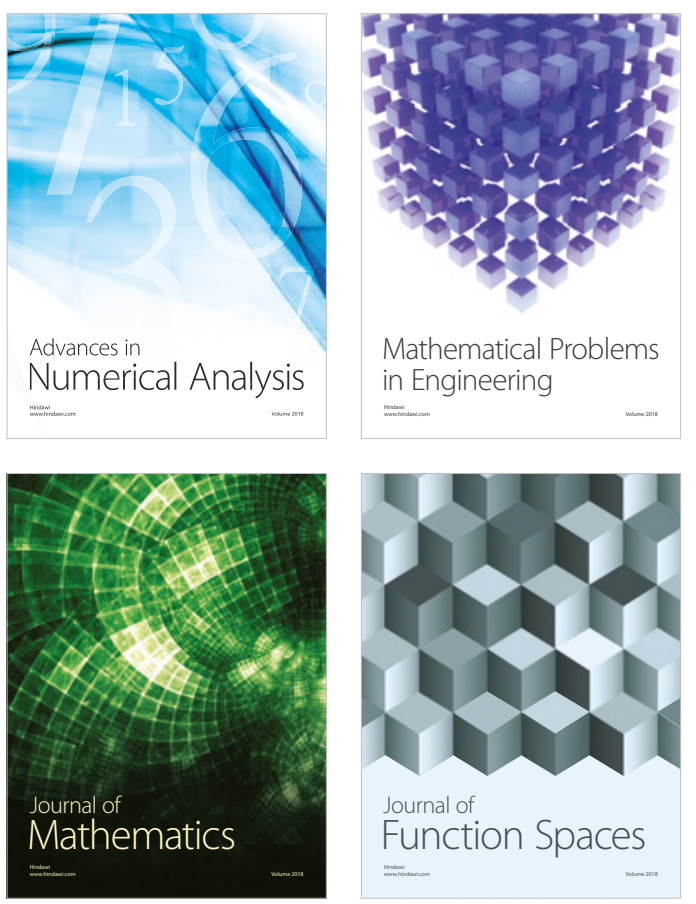

Mathematical Problems in Engineering

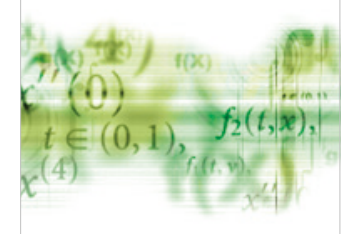

International Journal of

Differential Equations

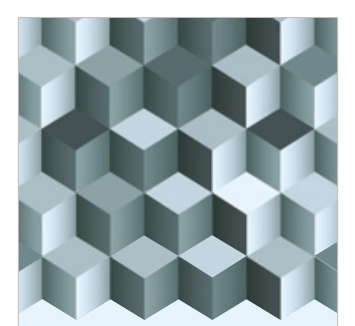

Journal of

Function Spaces
The Scientific

World Journal

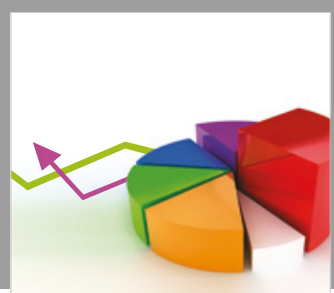

Journal of

Probability and Statistics
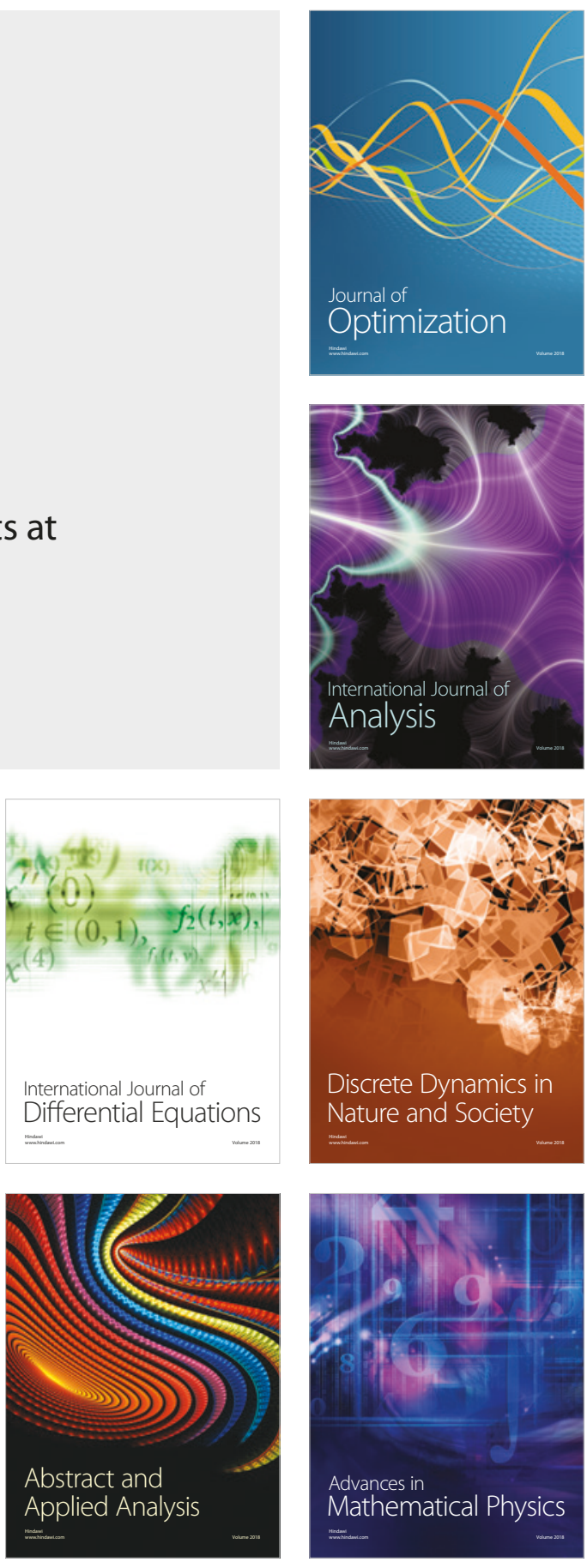